\title{
Teleradiotherapy of joints in rheumatoid arthritis: lack of efficacy
}

\author{
M Graninger, L Handl-Zeller, G Hohenberg, A Staudenherz, F Kainberger, W Graninger
}

Ann Rheum Dis 2005;64:138-140. doi: 10.1136/ard.2004.021766

Background: Low dose radiotherapy is commonly used for painful rheumatic conditions in clinical practice. Teleradiotherapy may be a cheap, painless procedure which is applicable to many joints at a time.

Objective: To determine if the local application of $x$ rays to inflamed joints in rheumatoid arthritis (RA) affects the signs and symptoms of inflammation.

Methods: In a randomised, controlled, double blind study, roentgen irradiation was administered in a total dose of 20 Gy during 2 weeks to single joints in six patients with RA who were receiving constant and stable pharmacological treatment with DMARDs and NSAIDs. Single inflamed joints on the contralateral side of the body were used as controls and received sham irradiation. Swelling and tenderness was assessed by blinded investigators before and until 3 months after the irradiation; general disease activity and pain scales were included in the assessment.

Results: No change in the scores for tenderness, swelling, pain, or disease activity was seen. The trial was stopped for ethical reasons.

Conclusion: Local roentgen treatment of RA at a substantial dose of 20 Gy was ineffective in this pilot trial.

$\mathrm{T}$ reatment of rheumatoid arthritis (RA) has been revolutionised recently by the advent of new immunomodulatory drugs, including tumour necrosis factor blockers and interleukin 1 antagonists. However, a high number of patients are refractory even to these new and expensive drugs so that further treatment strategies are still being sought.

An established intervention is the intra-articular application of radioactive substances (radiosynovectomy) to achieve necrosis of the inflamed synovia in accessible joints. ${ }^{1}$ However, injection of radionuclides into the joints is limited to a small number of joints and usually is performed in the larger joints only. In contrast, non-invasive application of radioablative irradiation doses (termed teleradiotherapy) would be cheap and advantageous technically, avoiding the risk of pain and bacterial contamination. This principle has been used previously for several painful rheumatic conditions. ${ }^{2-4}$ A double blind, placebo controlled trial of roentgen irradiation in periarthritis of the shoulder and osteoarthritis did not result in significant differences of pain. ${ }^{4}$

Only a few reports have investigated the efficacy of such teleradiotherapy in inflammatory arthritides..$^{5-8} \mathrm{~A}$ high success rate of local doses of 5-7 Gy was reported in comparison with laser therapy and concomitant local glucocorticoid injections. ${ }^{7}$ A study comparing unblinded radiosynovectomy, $x$ ray irradiation, and $\gamma$ irradiation did not contain an unirradiated group. ${ }^{8}$ We performed a placebo controlled study using a local electron beam applied to affected joints in patients with active RA.

\section{PATIENTS AND METHODS}

\section{Patients}

Six female patients with longstanding RA and persistent disease activity despite adequate use of disease modifying antirheumatic drugs (DMARDs) participated in this prospective study. After informed written consent, patients were included if they fulfilled the inclusion criteria of active rheumatoid arthritis (28 joint count Disease Activity Score (DAS28) >5.0, at least two swollen and tender joints), unsuccessful use of at least one DMARD, current use of methotrexate for at least 3 months, treatment of pain with full dose non-steroidal anti-inflammatory drugs. Exclusion criteria were age $<30$, inadequate means of birth control, pregnancy, prednisone doses $>10 \mathrm{mg} /$ day, injections into any joint during the past 3 months, any radiosynovectomy, and use of chloroquine, ${ }^{9}$ cyclophosphamide or azathioprine or tumour necrosis factor blocking agents. Table 1 lists the patient characteristics. The protocol was approved by the local ethical committee.

\section{Methods}

In each patient, two possible target joints were determined. The patients were referred to the department of radiation therapy for the planning of radiotherapy. A computer generated randomisation list was used by the radiation oncologist to determine which of the two joints was to receive the radiation; the other joint was treated by sham radiation. Neither patient nor rheumatologist were informed about the randomisation allocation. The selected joints were marked at the skin and the irradiated volume was simulated in such a way that only the anatomical joint including the joint capsule was treated.

Using a $20 \mathrm{MeV}$ linear accelerator, a total depth dose of 20 Gy was given in 10 fractions over 2 weeks to the target joint. For all patients, a very loud sound was given over headphones, so that the special noise accompanying radiation could not be perceived and sham treatment could not be distinguished.

Before and after radiation therapy, and 4 and 10 weeks thereafter, study visits were arranged with the patients. At each visit, a rheumatological examination was carried out, including routine laboratory assessment and determination of the number of swollen and tender joints, DAS28, ${ }^{10}$ and a Health Assessment Questionnaire score. In addition, the degree of joint swelling and tenderness was expressed on a 0 to 3 ordinal scale. All examinations were performed by the same physician, and neither patient nor rheumatologist knew

Abbreviations: DAS28, 28 joint count Disease Activity Score; DMARDs, disease modifying antirheumatic drugs; RA, rheumatoid arthritis 


\begin{tabular}{|c|c|c|c|c|c|}
\hline $\begin{array}{l}\text { Patient } \\
\text { No }\end{array}$ & Age & $\begin{array}{l}\text { Time since } \\
\text { diagnosis of } \\
\text { RA (years) }\end{array}$ & Current DMARD treatment & Irradiated joint & $\begin{array}{l}\text { Sham treated } \\
\text { joint }\end{array}$ \\
\hline 1 & 38 & 17 & MTX (10 mg /week)+SSZ $(2 \mathrm{~g} /$ day $)$ & MCP \| right & Shoulder left \\
\hline 2 & 39 & 19 & $\begin{array}{l}\text { Leflunomide ( } 20 \mathrm{mg} / \text { day })+M T X \\
(10 \mathrm{mg} / \text { week) }\end{array}$ & MCP II right & MCP II left \\
\hline 3 & 57 & 4 & MTX (20 mg/week) & Wrist right & Wrist left \\
\hline 4 & 66 & 17 & MTX (25 mg/week) & Knee left & Elbow right \\
\hline 5 & 61 & 10 & MTX (25 mg/week) & Knee left & PIP III right \\
\hline 6 & 61 & 16 & MTX $(10 \mathrm{mg} /$ week $)+\operatorname{SSZ}(2 \mathrm{~g} /$ day $)$ & $M C P \vee$ right & Ankle left \\
\hline
\end{tabular}

which of the joints received sham or placebo irradiation. Plain radiographs of the irradiated and sham treated joints were obtained at baseline, 3 months, and 1 year after the irradiation treatment.

In one patient nuclear magnetic resonance examinations of the treated joints were performed before and 3 months after treatment; in the same patient paired joints results of scintigraphies with human immunoglobulin were available. Local scans were performed 4 hours after the injection of $555 \mathrm{MBq}$ ( $15 \mathrm{mCi}$ ) of ${ }^{99 \mathrm{~m}}$ Tc-HIG (human immunoglobulin; TechneScan HIG, Mallinckrodt Medical BV, Holland) and reviewed by two observers who did not know the clinical, radiological, and therapeutic situation). Routine laboratory examinations were performed at each visit and included, erythrocyte sedimentation rate, $\mathrm{C}$ reactive protein, rheumatoid factor, antinuclear antibody, blood chemistry, and full blood count, as well as pregnancy tests in premenopausal women.

\section{RESULTS}

Fractionated local joint irradiation was well tolerated, and the patients did not report any adverse reactions. No clinically relevant change was found in any of the outcome measures. Figure 1 shows that only slight variations in the degree of swelling and tenderness were recorded in the irradiated joints, the change of values for the sham irradiated joints was similarly small. A statistical evaluation was not performed because an interim analysis after treating six patients showed no therapeutic effect of irradiation after 6 weeks, and the study had to be stopped for ethical reasons.

Neither disease activity nor health assessment showed any changes before and 3 months after treatment. Radiographs showed erosive disease without ankylosis in all treated joints, but no change throughout the observation period. In the one case where nuclear magnetic resonance and scintigraphy were available, the blinded observers did not report any change 3 months after sham treatment or irradiation. During
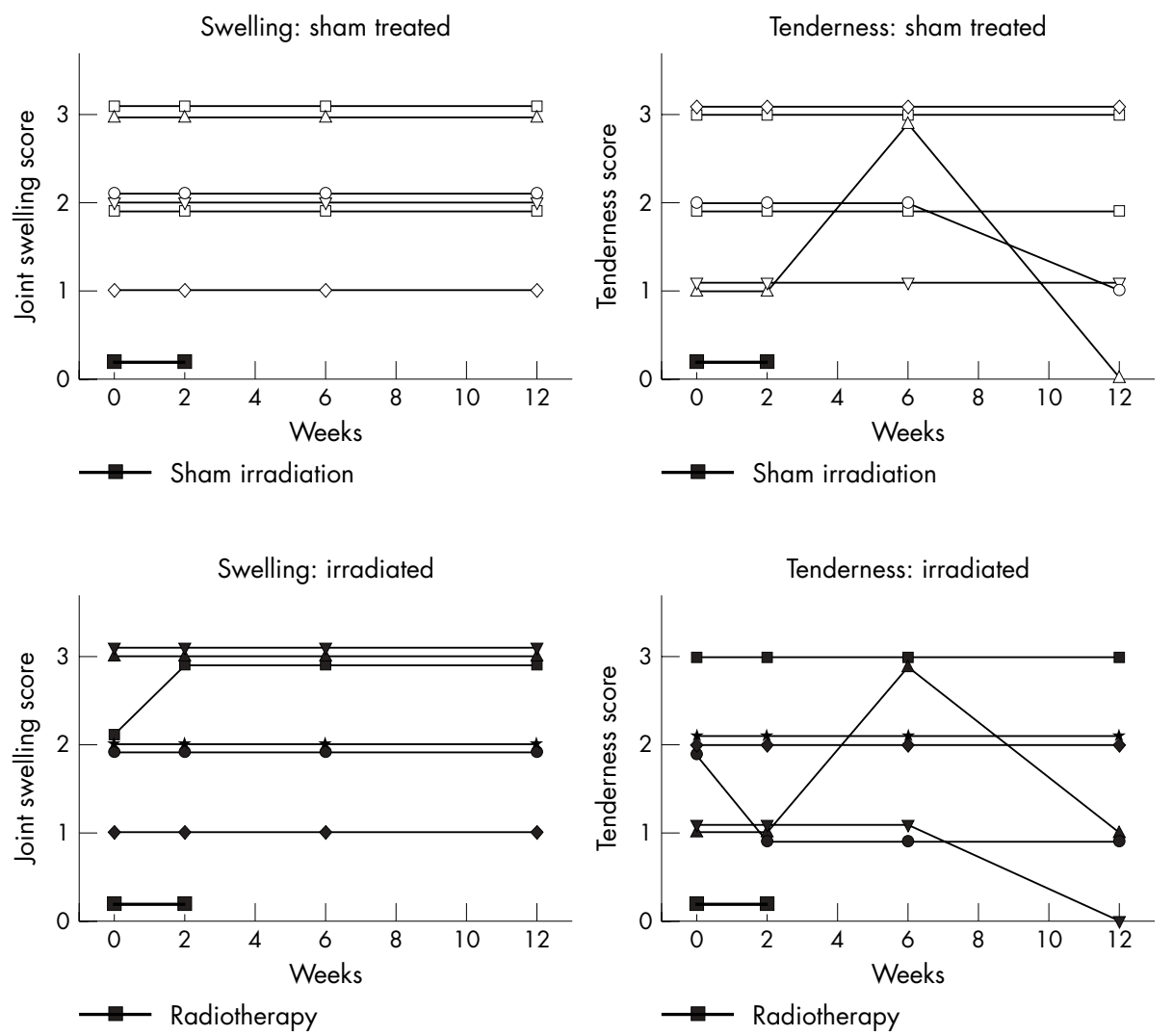

Figure 1 Results from joint assessment of irradiated and sham treated joints in six patients. Swelling and tenderness were rated by a blinded observer on an arbitrary scale from 0 to 3 . Dark bars indicate the period of 2 weeks during which the total dose of 20 Gy was administered. 
a control visit after 1 year, disease activity had improved in five of the six patients in whom an intensified DMARD regimen had been installed after termination of the study.

\section{DISCUSSION}

RA is characterised by painful swelling of the joint capsule (synovitis). The inflammatory infiltrate in the synovia and at the cartilage-pannus junction consists of activated fibroblasts, monocytes, and lymphocytes, which show histochemical signs of activation like HLA-DR expression, CD25 expression, and dysregulation of apoptosis. ${ }^{11}$ Induction of apoptosis or necrosis of these activated cells by irradiation with ionising radiation might theoretically be a successful way to control the activity of this destructive disease. In animal models of adjuvant induced arthritis, ${ }^{12}{ }^{13}$ such efficacy has been suggested.

Distant radiation therapy has been proposed as an effective treatment of RA in the previous years. ${ }^{5-8}$ The most recent study from Russia compared the efficacy of teleradiotherapy with that of radiosynovectomy, but did not include a placebo control. ${ }^{8}$ In contrast with these reports, our controlled study had to be stopped for lack of efficacy. These different results may be explained by the selection of patients and the differences in concomitant drug treatment, severity and duration of the disease, or the doses of radiation applied.

A limitation of our study design might be the selection of the total dose of irradiation. However, the dose we used was high; it was selected from experience with the treatment of heterotopic ossification, where doses of up to $17.5 \mathrm{~Gy}$ are used. ${ }^{14}$ As with other treatment approaches for RA, the effect of teleradiotherapy might be more beneficial earlier in the course of the disease. However, the observations from our pilot trial do not support the use of teleradiotherapy in RA.

\author{
Authors' affiliations \\ M Graninger, W Graninger, Department of Rheumatology, Vienna \\ University Medical School, Vienna, Austria \\ L Handl-Zeller, G Hohenberg, Department of Oncological \\ Radiotherapy, Vienna University Medical School, Vienna, Austria \\ A Staudenherz, Department of Nuclear Medicine, Vienna University \\ Medical School, Vienna, Austria
}

F Kainberger, Department of Radiology, Vienna University Medical School, Vienna, Austria

Correspondence to: Dr M Graninger, Department of Rheumatology, Internal Medicine III, General Hospital of Vienna, Währinger Gürtel 1820, A-1090 Vienna, Austria; monika.graninger@univie.ac.at

Accepted 7 April 2004

\section{REFERENCES}

1 Clunie G, Ell PJ. A survey of radiation synovectomy in Europe, 1991-1993. Eur J Nucl Med 1995;22:970-6.

2 Heyd R, Schopohl B, Bottcher HD. Radiation therapy in humero-scapular periarthropathy. Indication, method, results obtained by authors, review of the literature. Rontgenpraxis 1998;51:403-12.

3 Sautter-Bihl ML, Liebermeister E, Scheurig H, Heinze HG. Analgetic irradiation of degenerative-inflammatory skeletal diseases. Benefits and risks. Dtsch Med Wochenschr 1993;118:493-8.

4 Valtonen EJ, Lilius HG, Malmio K. The value of roentgen irradiation in the treatment of painful degenerative and inflammatory musculoskeletal conditions. A double-blind study. Scand J Rheumatol 1975;4:247-9.

5 Benenson EV, Ivanova RL, Ovsiannikova RS, Alibekova RI, Andreiashchenko NF. Assessment of the clinical effectiveness and mechanism of action of $x$-ray therapy in rheumatoid arthritis. Vopr Revm 1981:28-30.

6 Ivanova RL, Andreiashchenko NF, Benenson EV. X-ray therapy effectiveness in rheumatoid arthritis. Med Radiol (Mosk) 1981;26:25-7.

7 Ignat'ev VK. The local x-ray therapy of patients with rheumatoid arthritis. Ter Arkh 1994;66:47-9.

8 Marusenko IM, Ignat'ev VK, Zdorov AE. Local radiotherapy in combined treatment of rheumatoid arthritis. Ter Arkh 2001;73:15-19.

9 Utley JF, Sachatello CR, Maruyama Y, Avila J, King R. Radiosensitization of normal tissue by chloroquine. Radiology 1977;124:255-7.

10 Prevoo ML, van't Hof MA, Kuper HH, van Leeuwen MA, van de Putte $L B$, van Riel PL. Modified disease activity scores that include twenty-eight-joint counts. Development and validation in a prospective longitudinal study of patients with rheumatoid arthritis. Arthritis Rheum 1995;38:44-8.

11 Chou CT, Yang JS, Lee MR. Apoptosis in rheumatoid arthritis-expression of Fas, Fas-L, p53, and Bcl-2 in rheumatoid synovial tissues. J Pathol $2001 ; 193: 110-16$.

12 Hildebrandt G, Radlingmayr A, Rosenthal S, Rothe R, Jahns J, Hindemith M, et al. Low-dose radiotherapy (LD-RT) and the modulation of iNOS expression in adjuvant-induced arthritis in rats. Int J Radiat Biol 2003;79:993-1001.

13 Trott KR, Parker R, Seed MP. The effect of $x$-rays on experimental arthritis in the rat. Strahlenther Onkol 1995;171:534-8.

14 Seegenschmiedt MH, Keilholz L, Martus P, Goldmann A, Wolfel R, Henning F, et al. Prevention of heterotopic ossification about the hip: final results of two randomized trials in 410 patients using either preoperative or postoperative radiation therapy. Int J Radiat Oncol Biol Phys 1997;39:161-71. 